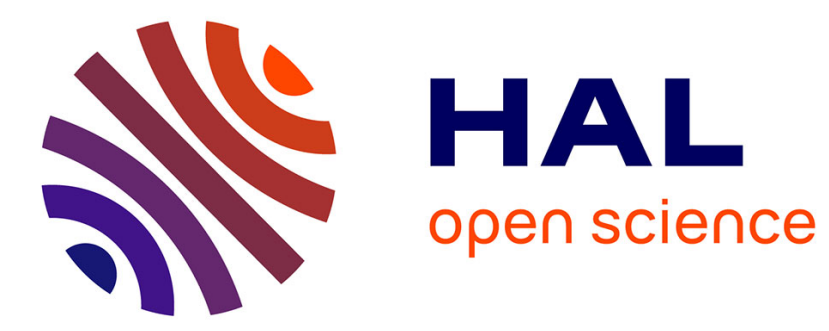

\title{
Modèle de sédimentation au passage Cénomanien/Turonien pour la formation Bahloul en Tunisie
}

Mohamed Soua, Nicolas Tribovillard

\section{- To cite this version:}

Mohamed Soua, Nicolas Tribovillard. Modèle de sédimentation au passage Cénomanien/Turonien pour la formation Bahloul en Tunisie. Comptes rendus de l'Académie des sciences. Série IIa, Sciences de la terre et des planètes, 2007, 339 (10), pp.692-701. 10.1016/j.crte.2007.08.002 . hal-00493062

\section{HAL Id: hal-00493062 \\ https://hal.science/hal-00493062}

Submitted on 20 Jun 2010

HAL is a multi-disciplinary open access archive for the deposit and dissemination of scientific research documents, whether they are published or not. The documents may come from teaching and research institutions in France or abroad, or from public or private research centers.
L'archive ouverte pluridisciplinaire HAL, est destinée au dépôt et à la diffusion de documents scientifiques de niveau recherche, publiés ou non, émanant des établissements d'enseignement et de recherche français ou étrangers, des laboratoires publics ou privés. 


\title{
Modèle de sédimentation au passage Cénomanien-Turonien pour la formation Bahloul en
} Tunisie

\section{Depositional model at the Cenomanian-Turonian boundary for the Bahloul Formation, Tunisia}

\author{
Mohamed Soua $^{1} \&$ Nicolas Tribovillard ${ }^{2}$
}

1 Entreprise Tunisienne d'Activités Pétrolières ETAP - CRDP, 4 Rue des Entrepreneurs. La Charguia II, 2035, Tunisia,

Courrier électronique : elmohology@yahoo.fr

2 Université Lille 1, Sciences de la Terre, UMR 8110 et FR1818, batiment SN5, F-59655 Villeneuve d'Ascq cedex, France. Courrier électronique : Nicolas.Tribovillard@univ-lille1.fr

\section{Résumé}

Plusieurs études multidisciplinaires ont été effectuées sur les couches riches en matière organique (MO) de la Formation Bahloul. Nos études de terrain et les synthèses géologiques, géochimiques, isotopiques et minéralogiques disponibles ont permis d'apporter des précisions nouvelles d'ordre paléogéographique et paléocéanographique. Ces données ont contribué à l'élaboration d'un modèle de dépôt sous une zone à minimum d'oxygène (ZMO) dans des bassins marins profonds et subsidents de type bathyal, où se sont accumulées et préservées d'importantes quantités de MO (jusqu'à 18,7\% de COT). Le présent modèle a permis la corrélation de la Formation Bahloul à d'autres coupes de référence à l'échelle globale, ce qui permet de mettre en évidence que le $d^{13} \mathrm{C}$ mesuré sur MO serait un meilleur outil de corrélation que le $d^{13} \mathrm{C}$ mesuré sur carbonates pour reconnaître la limite Cénomanien/Turonien.

Mots clés Formation Bahloul ; Géochimie ; Stratigraphie ; EAO-2 ; Cénomanien-Turonien ; matière organique; modèle de dépôt.

\begin{abstract}
Several multidisciplinary studies were undertaken on the Cenomanian-Turonian organic-rich Bahloul Formation. These studies, as well as our new data, led to some new paleogeographic and paleoceanographic interpretations. Based on these data, a depositional model was elaborated pointing out that at the $\mathrm{C} / \mathrm{T}$ transition an oxygen minimum zone (OMZ) impinged onto the south Tethyan margin through the Tunisian intrashelf basins formed during early Cretaceous. This model allows correlating the Bahloul Fm. to other OAE-2 formations throughout the world. It also indicates

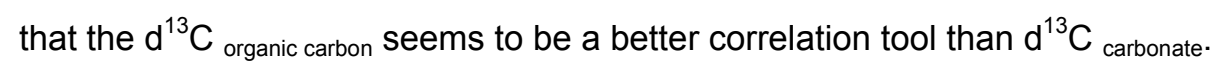

Keywords Bahloul Formation; geochemistry; stratigraphy; OAE-2; Cenomanian-Turonian; Organic matter; Depositional model.

Abridged English version 
During the late Cenomanian-early Turonian Oceanic Anoxic Event (OAE-2 or Bonarelli event), organic-rich black shales were deposited worldwide coinciding with the strong transgressive period during uppermost Cenomanian. New ecological niches were created and favoured intense biological proliferation that induced a massive organic matter (OM) production in surface water. An oxygen minimum zone (OMZ) impinged onto the South Tethyan margin, where the organic-rich Bahloul Formation (Fig.1A) is the expression of the OAE-2 event in North-Central and South-central Tunisia. Consequently, a great quantity of OM has accumulated in different basinal environments $[1,2,3,6,7$, $20,21,22]$. The sealing of lower Cretaceous structures, during distensif regime has persisted and formed graben systems that promoted organic-rich strata deposition throughout upper Cenomanian to lowermost Turonian times (Fig.2).

The paleogeographic interpretation suggests that the Bahloul facies distribution (Fig.1A) is limited to the intermediate deep water mass and could correspond to an $\mathrm{OMZ}$ environment of sedimentation (Fig. 2) towards the South Tethyan margin [3]. Generally 20 to $30 \mathrm{~m}$ thick in average, the Bahloul deposits are composed of dark laminated limestones alternating with light grey indurated marls (Fig.3). This alternation is considered as the expression of a climatic Milankovitch-type cyclicity recording 20 ky precession [11] that correlates with $\mathrm{CaCO}_{3}$ fluctuations. These deposits correspond to high TOC values reaching 18,7 \% [1]. The Bahloul was deposited during the uppermost Cenomanian (upper part of the Rotalipora cushmani Zone) to the lowermost Turonian (either top Whiteinella archaeocretacea Zone or base of Helvetoglobotruncana helvetica Zone) and corresponds largely to a basal transgressive system tract (TST). All the studied sections in Tunisia, show that its base belongs to either a lowstand system tract (LST) or shelf margin wedge (SMW) [16, 21, 24].

A triple subdivision (referred to as peaks I, II and III) was based on small variations in the C-isotopic profiles that develop in the majority of the isotopic curves $\left(d^{13} \mathrm{C}\right)$ of all the studied sections and are pointed out by several authors [2, 3, 4, 23]. In all the studied sections, except for the Gafsa area where no foraminiferal studies were performed, the first isotopic excursion (I) always precedes the specialized keeled rotaliporids LO (Fig.3, Fig.4). The second isotopic excursion (II) generally occurs above the last occurrence (LO) of Rotalipora cushmani and the third one (III) just below the appearance of the Quadrum gartneri nannofossil zone marker (Fig.4). The Cenomanian - Turonian boundary occurs usually slightly above the maximum $d^{13} \mathrm{C}$ values [17]. Our results also indicate that

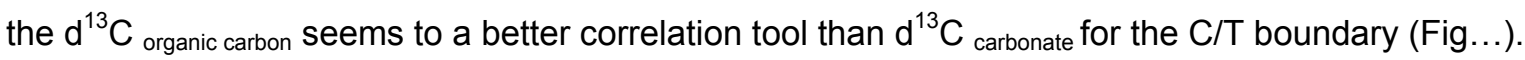
Nannofossils assemblages $[8,23]$ are characterized by a low diversity assigned to a combination of hostile conditions and dissolution during the Bahloul deposition. The paleoecologic data suggest that the $\mathrm{C} / \mathrm{T}$ deposits in Tunisia were restricted to a bathymetric belt which corroborates the installation of a small OMZ in North-central Tunisia and locally in the Gafsa Basin (Fig.5). This OMZ is marked by decline in the planktonic foraminiferal diversity [27]. These forms are mainly represented by globular forms (Guembelitria, Whiteinella, Heterohelix, Hedbergella). The organic-rich Bahloul provinces are characterized by a type-II marine kerogen (IH between 200 and $700 \mathrm{mg} \mathrm{HC} / \mathrm{g} \mathrm{TOC}$ ) [20] and TOC values reaching $18,7 \%$ [1], indicating excellent hydrocarbons source rock-quality. The OM thermal maturity presents a rather homogeneous distribution with $T_{\max }$ ranging between 430 and $500{ }^{\circ} \mathrm{C}$ (Fig.1B). 
In this synthesis dealing with the worldwide OAE-2 expression in the southern Tethyan margin, i.e. the Bahloul Fm., the organic-rich $\mathrm{C} / \mathrm{T}$ deposits are distributed into three different areas (Fig.1A) corresponding to small paleobasins sealed by several paleohighs (Fig.2). The established $d^{13} \mathrm{C}$ isotopic triple subdivision may be used as a high resolution correlation tool in a global scale (Fig.4). The depositional model carried out in this work shows that the OM distribution follows existing paleogeography during $\mathrm{C} / \mathrm{T}$ interval (Fig. 5).

\section{Introduction}

Les événements anoxiques océaniques (EAO) représentent des intervalles de stockage du carbone organique à l'échelle globale. Le terme EAO, proposé par Schlanger et Jenkyns [25] s'applique aux épisodes de distribution globale des black shales, laminés et riches en matière organique (>1\% pondéral de carbone organique total ou COT), enregistrés dans des séquences pélagiques de la partie médiane du Crétacé et plus particulièrement, celui d'âge Cénomanien - Turonien EAO-2 ou Bonarelli event $[19,25,26]$. Les épisodes majeurs de stockage de matière organique (MO) en domaine marin retentissent sur la composition isotopique du $\mathrm{C}$ de l'eau de mer qui conditionne à son tour la composition isotopique des substances qui s'y forment: carbonates et MO marine [2, 13]. En l'occurrence, I'EAO-2 correspond dans plusieurs coupes à travers le domaine téthysien à une augmentation du $\mathrm{d}^{13} \mathrm{C}$, observée sur les carbonates (+ $2,5 \%$ en moyenne) comme sur la $\mathrm{MO}(+4 \%$ en moyenne) [2, 18, 17, 26, 28]. Le calibrage biostratigraphique de la courbe représentative des variations isotopiques de $\mathrm{d}^{13} \mathrm{C}$ confère à cette anomalie positive $[2,17,26]$ un caractère synchrone susceptible d'être utilisé pour des corrélations à grandes distances [2, 18, 26, 28]. Les données isotopiques de $\mathrm{d}^{13} \mathrm{C}$ couplées à un calage biostratigraphique et à des données de $\mathrm{COT}$ et de $\mathrm{CaCO}_{3}$ nous ont permis d'affiner la corrélation des dépôts du Cénomanien-Turonien $(\mathrm{C} / \mathrm{T})$ du faciès Bahloul (Tunisie) à partir des travaux antérieurs. Le but de cet article est de montrer d'une part l'existence d'une zone à minimum d'oxygène (ZMO) régnant lors du dépôt des black shales du faciès Bahloul et, d'autre part, de proposer un modèle de dépôt rendant compte de leurs caractères, pour une région de la Téthys ayant bien enregistré la sédimentation du Crétacé supérieuret notamment la limite Cénomanien/Turonien (C/T).

\section{Relation entre l'accumulation de la MO et la structuration de la Tunisie au Cénomanien- Turonien}

Le cadre géologique de la Tunisie montre deux domaines distincts (Fig. 1) : (1) une zone subsidente située au nord, formée de terrains mésozoïques et cénozoïques épais, et caractérisée par des déformations alpines, et (2) une province paléozoïque stable située plus au sud, occupée par des terrains jurassiques et par des structures plus jeunes, relativement minces et non déformées $[7,10]$. Les couches, d'âge $\mathrm{C} / \mathrm{T}$ riches en $\mathrm{MO}$, ne sont reconnues que dans le premier domaine structural (Fig.1A). La distribution et le contenu organique de ce faciès ont été fortement contrôlés par l'emplacement des fossés d'effondrement durant la transition $C / T$ [1, 5, 6, 7, 20, 21]. En revanche, pour quelques régions et durant la même période, les interprétations privilégient des déplacements verticaux pour expliquer les mouvements ascensionnels synsédimentaires du complexe triasique [1, 
7]. La structuration de ces fossés, liés à la phase de rifting, est principalement associée à l'ouverture de la Néotéthys vers le Nord-Est [15] et à celle de l'Atlantique central au Sud-Ouest. Cette phase de rifting en Tunisie n'a pas commencé avant la période Jurassique supérieur - Crétacé inférieur $[5,7$, 14, 15]. Le rifting enregistré au cours du Trias - Jurassique inférieur serait plutôt en liaison avec la séparation du bloc Apulien lié, à cette période, au bord septentrional de la plaque africaine [14]. Le maximum de la richesse en $\mathrm{MO}$ des dépôts $\mathrm{C} / \mathrm{T}$ est habituellement atteint dans la périphérie des dômes triasiques mais surtout dans les fossés d'effondrement (Fig. 2). Les mouvements diapiriques ont également joué un rôle en contrôlant les dépôts $\mathrm{C} / \mathrm{T}$ en Tunisie septentrionale.

\section{Extension et caractéristiques du faciès Bahloul en Tunisie}

Durant l'intervalle $\mathrm{C} / \mathrm{T}$, la Tunisie se situait dans la marge sud de la Téthys dont elle était limitée par un talus pendant généralement vers le Nord (Fig.5). Dans ce domaine, se sont accumulés des faciès riches en MO, regroupés sous le nom de Formation Bahloul par P.-F. Burollet [9]. L'existence d'une telle province riche en $\mathrm{MO}$ a été confirmée par des données pétrolières $[5,6,7]$ et d'affleurement $[1,3$, $20,21,23,24,27]$. L'interprétation paléogéographique (Fig.1A) suggère que la distribution du faciès Bahloul ait été limitée à la profondeur de la tranche d'eau intermédiaire (quelques centaines de mètres), sous f'influence d'une ZMO vers la marge sud de la Téthys [3]. Épais de 20 à 30 mètres en moyenne, le Bahloul est formé d'une alternance régulière de calcaires laminés et/ou noduleux noirs à grisâtres et de niveaux marneux présentant des valeurs de COT qui oscillent entre $1 \%$ et $13,35 \%$ [4]. Une valeur de 18,7\% de COT [1] a même été rapportée dans un échantillon du bassin de Gafsa (Oued Berda). Ces alternances de faciès, plus ou moins riches en MO (Fig. 3), sont interprétées comme résultant d'alternances d'oxygénation des fonds marins, elles-mêmes interprétées comme le résultat de cycles de précession de $20 \mathrm{ka} \mathrm{[11].} \mathrm{Elles} \mathrm{existent} \mathrm{également} \mathrm{sur} \mathrm{une} \mathrm{échelle} \mathrm{plus} \mathrm{petite,}$ sous forme d'une alternance entre des lamines claires et des lamines sombres d'échelle millimétrique. La plupart des coupes étudiées montrent une augmentation rapide de la valeur de COT à la base, reflétant la transition de conditions aérobies à des conditions anaérobies [23]. En Tunisie, au sein du Bahloul, a été mis en évidence un faciès riche en MO comprenant plusieurs intercalations de couches riches en radiolaires [27] signalé aussi en Algérie, au nord de l'accident sud atlasique, en plusieurs localités [10] formant probablement une bande continue le long de la plateforme nord africaine au cours de la partie moyenne du Crétacé en allant de la Tunisie jusqu'au Pré-Rif marocain [23]. De tels dépôts siliceux riches en MO ont été définis en 1986 comme étant le marqueur de la limite C/T [28].

\section{Age et séquences du Bahloul}

La formation Bahloul s'est déposée durant le Cénomanien supérieur (partie supérieure de la zone à Rotalipora cushmani) et le Turonien inférieur (partie inférieure de la zone à Helvetoglobotruncana helvetica). La partie supérieure de cet horizon est connue pour être diachrone. Son âge s'étend donc sur toute la zone à $W$. archaeocretacea jusqu'à la partie basale de la zone à Helvetoglobotruncana helvetica. Malgré les recherches menées dans des secteurs miniers tels que Bougrine et Garn Halfaya, où un âge $C / T$ a été attribué par calage stratigraphique [22], le Bahloul dans la région de Kalâat Senan était supposé cénomanien supérieur [24]. Cependant, cette interprétation a été plus tard 
corrigée par Accarie et al. [2] qui, avec les moyens biostratigraphiques fournis par les nannofossiles calcaires, ont démontré que la Formation Bahloul dans cette région atteint également le Turonien inférieur, conformément aux idées de Maamouri et al. [21]. La durée minimale du dépôt de ce faciès riche en $\mathrm{MO}$ dans la région de Gafsa s'étend depuis la partie inférieure de la zone à ammonite à Metoicoceras geslinianum jusqu'à la partie moyenne de la zone à Pseudaspidoceras flexuosum [1]. Le Bahloul en Tunisie coïncide généralement avec un intervalle transgressif (IT) enregistrant des valeurs de COT relativement élevées. Dans quelques régions, sa partie basale peut appartenir au prisme de bas niveau marin (PBN), comme dans la région de Guern Halfaya [21] ou à un prisme de bordure de plateforme (PBP) comme dans la coupe type [21] et la région de Kalâat Senan [24]. Le prisme de haut niveau (PHN) surmontant I'IT à cachet Bahloul dans la plateforme interne tunisienne est généralement représenté par des dépôts de faible tranche d'eau, comme les carbonates marins bioclastiques du Gattar ou les unités équivalentes, alors que le faciès le plus septentrional est représenté par les argiles de la Formation Kef.

\section{Caractéristiques isotopiques du Bahloul}

La composition isotopique du C a été analysée, parfois avec une forte résolution, dans de nombreuses coupes du Bahloul $[2,3,13]$. Nous avons tenté de corréler ces données entre les différentes coupes, dans le but de comparer la distribution $d u d^{13} \mathrm{C}$ à l'échelle tunisienne et de comparer ces courbes du d ${ }^{13} \mathrm{C}$ avec les données existant à l'échelle mondiale [26]. Notamment, nous avons tenté d'identifier les trois pics ou "excursions" positives du d ${ }^{13} \mathrm{C}$ observés dans plusieurs coupes de la limite C/T (Western Interior, Bassin Anglo-parisien en particulier [8, 26]). Dans ce but, la corrélation établie a dû être étayée par un calage biostratigraphique fin (foraminifères planctoniques, nannofossiles et ammonites). La figure 3 montre que dans plusieurs coupes tunisiennes, on peut reconnaître les trois pics (dénommés ici I, II et III) avec les mêmes calages biostratigraphiques caractéristiques (positions relatives par rapport à la dernière occurrence de Rotalipora cushmani et apparition du nannofossile marqueur Quadrum gartneri). Cependant, les onze coupes tunisiennes montrent que les trois pics sont beaucoup plus visibles lorsqu'on dispose de la composition isotopique du C mesurée sur la MO plutôt que sur les carbonates. Les variations du réservoir océanique de $C$ seraient ainsi mieux enregistrées par la MO d'origine marine que par les carbonates formés en milieu marin. En Tunisie, on n'observe pas de covariations précises entre les courbes de $d^{13} \mathrm{C}$, COT et $\mathrm{CaCO}_{3}$, mais on remarque que, globalement, les valeurs de COT sont plus fortes dans l'intervalle de plus fortes valeurs de $d^{13} \mathrm{C}$, avec un décalage temporel (retard) dans l'élévation des valeurs de COT. Ce phénomène est observé ailleurs également dans le monde [3, 18].

La figure 3 montre une absence de co-variation entre la distribution du TOC et celle du $\partial 13 \mathrm{C}$ (mesuré sur matière organique ou carbonates). La quantité de matière organique accumulée dans les sédiments dépend de nombreux paramètres (nature des organismes sources, intensité de la production, épaisseur de la colonne d'eau, taux de sédimentation, granularité des sédiments, degré d'oxygénation de la colonne d'eau, caractère redox des eaux interstitielles, etc. [29]). Le contenu en carbone organique (COT) est donc un paramètre qui dépend énormément des conditions locales de sédimentations. II ne peut donc guère être utilisé comme outil de corrélation à une échelle fine (même 
si certains épisodes de l'histoire de la Terre, à l'instar de la limite $\mathrm{C} / \mathrm{T}$, sont identifiables par un COT élevé). En revanche, le $\partial 13 \mathrm{C}$ (organique ou minéral) est un paramètre lié au réservoir océanique de carbone et relativement peu sensible aux conditions locales de sédimentation ; sa valeur d'outil de corrélation est donc bien connue pour être bonne. Pour conclure, nos résultats montrent que si la limite $\mathrm{C} / \mathrm{T}$ peut être facilement identifiée à l'échelle téthysienne par la mesure du COT, il faut avoir recours au $\partial 13 \mathrm{C}$ pour les corrélations fines à l'échelle de cette limite.

\section{Caractéristiques paléoécologiques}

Les recherches paléoécologiques détaillées réalisées dans les dépôts du Bahloul ont utilisé l'analyse des microfaciès [24], le caractère planctonique/benthique des foraminifères [27], les nannofossiles calcaires [2, 8, 23] et les ammonites [1]. Les assemblages des nannofossiles sont caractérisés par une faible diversité attribuée à une combinaison de conditions hostiles et une dissolution durant la sédimentation [8, 23]. Un renouvellement faunique dans les assemblages des nannofossiles a été enregistré dans la Formation Bahloul où la forme opportuniste Watznaueria barnesae représente l'espèce la plus fréquente $[8,23]$. Les données paléoécologiques suggèrent que les dépôts $C / T$ sont limités par une ceinture bathymétrique [27] qui peut refléter l'installation d'une petite ZMO en Tunisie centro-septentrionale et, localement, dans le bassin de Gafsa. Cette ZMO est marquée par l'abondance des Heterohelix dès la base de la formation et la réduction des formes planctoniques chez les foraminifères qui ne sont plus représentés que par des formes à loges globuleuses (Guembelitria, Whiteinella, Hedbergella) et l'absence des formes benthiques [27]. Toutefois, la préservation des ammonites et la présence de niveaux repères régionaux à bioturbations évoquent un environnement alternativement anoxique et dysoxique. Les habitants de subsurface de la zone photique inférieure perdent leurs habitats suite à l'expansion de cette ZMO et disparaissent. Le début du rétrécissement de cette zone à la fin du haut niveau eustatique a peut-être créé de nouveaux habitats induisant le renouvellement faunique enregistré chez les radiolaires et les foraminifères planctoniques $[12,27]$.

\section{Caractéristiques géochimiques de la MO du Bahloul}

Les provinces où la formation Bahloul est riche en $\mathrm{MO}$ sont caractérisées par un kérogène d'origine marine de type II (IH entre 200 et $700 \mathrm{mg} \mathrm{HC} / \mathrm{g} \mathrm{COT}$ ) avec des concentrations en COT de plus de $10 \%$, indiquant de roches mères d'hydrocarbures d'excellentes qualités $[3,7,20,22]$. La maturité thermique de la $\mathrm{MO}$ présente une distribution variable avec des $T_{\max }$ généralement compris entre 430 et $500{ }^{\circ} \mathrm{C}$ mais pouvant atteindre localement des valeurs plus fortes(Fig.1B). L'observation au microscope de ces sédiments indique que le kérogène est dominé par une matière organique amorphe (MOA). Son origine algaire ou bactérienne se manifeste par une forte fluorescence jaunâtre sous lumière ultraviolette. La distribution des biomarqueurs moléculaires est caractérisée par des $n$ alcanes, des stéranes, des hopanes et des isoprénoïdes acycliques indiquant une MO d'origine marine (algaire ou zooplanctonique) [3]. Les méthylhopanes indicateurs de cyanobactéries ou de méthylotrophes sont largement présents dans les dépôts de la formation Bahloul [3]. L'occurrence du 
bisnorhopane dans ces black shales reflète une source plutôt bactérienne probablement liée à un environnement de dépôt fortement réducteur riche en soufre.

\section{Discussion sur les environnements de dépôts}

\subsection{Relation entre paléogéographie et expansion d'une ZMO : modèle de dépôt}

La tectonique distensive qui a favorisé l'ouverture de plusieurs bassins (Fig. 2) a permis localement le dépôt de sédiments riches en MO sous une ZMO. Ces petits bassins sont limités par des paléo-hautsfonds (Fig. 5) qui ont peut-être limité les échanges des eaux océaniques déjà appauvries en oxygène en induisant une stratification des eaux marines. Ces paléoreliefs ont réagi à des manifestations diapiriques ayant provoqué des inversions de subsidence marquant des irrégularités dans la topographie.

La distribution de la MO reflète la paléogéographie existant au cours de l'intervalle $\mathrm{C} / \mathrm{T}$ avec des zones relativement hautes qui étaient baignées par une ZMO où la circulation réduite a engendré un déficit en oxygène (Fig. 5). Un autre mécanisme applicable pour le bassin de Gafsa est la mise en place d'une halocline ayant provoqué la stratification des eaux [1]. Cette dernière fut causée par la circulation des eaux hypersalées engendrées par le lessivage des évaporites du Trias.

\subsection{Les zonations de maturité de la Formation Bahloul}

Dans la majorité des sites étudiés en Tunisie (Fig. 1B), la MO du Bahloul [3, 20, 22] a connu une évolution diagénétique assez poussée (domaine de la catagenèse). La distribution du Bahloul se répartit en couloirs d'orientation préférentielle NE-SW dans le domaine centro-septentrional et d'orientation presque E-W à NE-SW dans le domaine oriental. La MO contenue dans ce faciès montre divers stades de maturité : (1) immature $\left(425\right.$ à $\left.435^{\circ} \mathrm{C}\right)$ à submature $\left(435^{\circ} \mathrm{C}\right)(2)$ mature $(435$ à $450^{\circ} \mathrm{C}$ ) et (3) Surmature $\left(450\right.$ à $550^{\circ} \mathrm{C}$ ). Ces variations des $T_{\max }$ et de maturité reflètent la subsidence et le flux thermique qui ont contrôlé la maturation de la MO.

\subsection{Contexte global}

L'examen de la $\mathrm{MO}$ et des profils isotopiques de $\mathrm{d}^{13} \mathrm{C}$ des coupes $\mathrm{C} / \mathrm{T}$ dans plusieurs localités du monde a montré que les extinctions majeures de la faune se sont produites pendant des intervalles de temps caractérisés par des valeurs de $d^{13} \mathrm{C}$ relativement élevées (Fig. 3). Ces valeurs isotopiques élevées coïncident généralement avec le dépôt de black shales dominés par une $\mathrm{MO}$ d'origine marine (kérogène de type II). Les marqueurs isotopiques, paléoécologiques, biostratigraphiques et géochimiques dont nous disposons ici peuvent être des outils de corrélation à l'échelle mondiale (Fig. 4). Les trois excursions majeures et significatives du profil isotopique de $d^{13} \mathrm{C}$ sont trouvées dans la majorité des coupes C/T étudiées en Tunisie (Fig. 3) et dans le monde (Fig. 4). Ces trois pics nous permettent de bien corréler les bassins lointains isolés. La similitude paléoécologique et l'extinction simultanée de la faune à formes spécialisées (foraminifères, radiolaires, ammonites, nannofossiles calcaires...) survenant pendant l'intervalle séparant la première et la troisième excursion de $d^{13} C$, nous permettent de bien localiser et reconstituer le scénario de l'événement anoxique EAO-2. Par ailleurs, notre interprétation, en se basant sur les premières occurrences ( $\mathrm{PO}$ ) et dernières 
occurrences (DO) des nannofossiles calcaires et des foraminifères, montre que cet événement n'est pas synchrone (Fig. 4) à l'échelle mondiale.

\section{Conclusion}

De cette synthèse, il ressort le fait que les dépôts $\mathrm{C} / \mathrm{T}$ riches en $\mathrm{MO}$ du Bahloul se répartissent en trois domaines différents correspondant à des emplacements de paléobassins profonds qui montrent des paléoreliefs différents. Ces emplacements ont été le siège de dépôts de sédiments de type bathyal dont l'âge varie de la partie supérieure de la zone à Rotalipora cushmani (Cénomanien supérieur) jusqu'au toit de la zone à Whiteinella archaeocretacea et dans quelques régions jusqu'au mur de la zone à Helvetoglobotruncana helvetica. Ces black shales, marquant l'événement d'inondation le plus intense au cours du Phanérozoïque, ont été déposés dans un intervalle transgressif. Une corrélation des données isotopiques du carbone complétée par des résultats géochimiques et par des données biostratigraphiques a permis une subdivision fournissant un outil de corrélation à l'échelle régionale, compatible avec ce qui est observé à l'échelle globale. Le modèle de dépôt proposé ici indique que la distribution de la MO suit la paléogéographie existant au cours de l'intervalle $\mathrm{C} / \mathrm{T}$ avec des zones relativement hautes baignées par une ZMO où la circulation réduite a peut-être fait augmenter le déficit en oxygène. Toutefois, la préservation des ammonites et la présence de niveaux repères régionaux à bioturbations dans cet intervalle de temps évoquent un environnement alternativement anoxique et dysoxique.

Nous soulignons qu'il ressort de l'analyse des séries tunisiennes que le $d^{13} \mathrm{C}$ marque des évolutions beaucoup plus tranchées quand il est mesuré sur la $\mathrm{MO}$, par rapport aux valeurs obtenues sur carbonates. De ce fait, les excursions $d u d^{13} \mathrm{C}$ sont beaucoup plus aisément corrélables. Ces observations suggèrent que le $d^{13} \mathrm{C}$ mesuré sur MO serait un meilleur outil que le $d^{13} \mathrm{C}$ mesuré sur carbonates pour déterminer la limite $\mathrm{C} / \mathrm{T}$.

\section{Références bibliographiques}

[1] H. Abdallah, S. Sassi, C. Meister et R. Souissi, Stratigraphie séquentielle et paléogéographie à la limite Cénomanien-Turonien dans la région de Gafsa-Chotts (Tunisie centrale). Cretac. Res. 21, (2000) pp. 35-106

[2] H. Accarie, L. Emmanuel, F. Robaszynski, F. Baudin, F. Amédro, M. Caron and J.-F. Deconinck, La géochimie isotopique du carbone $\left(\mathrm{d}^{13} \mathrm{C}\right)$ comme outil stratigraphique. Application à la limite Cénomanien/Turonien en Tunisie centrale. C. R. Acad. Sci., Paris 322 lla, (1996) pp. 579-586.

[3] P. Barrett, A comparative organic geochemical and stable isotope study of the CenomanianTuronian organic-rich sediments from Tunisia, Germany and the UK. 1998 PhD thesis, University of Newcastle. $250 p$.

[4] A. Bechtel, M. Pevaz et W. Püttmann, Role of organic matter and sulphate-reducing bacteria for metal sulphide precipitation in the Bahloul Formation at the Bou Grine $\mathrm{Zn} / \mathrm{Pb}$ deposit (Tunisia). Chem. Geol. 144, (1998) pp. 1-21. 
[5] M. Bédir, N. Boukadi, S. Tlig, F.B. Timzal, L. Zitouni, R. Alouani, F. Slimane, C. Bobier and F. Zargouni - Subsurface Mesozoic basins in the central Atlas of Tunisia: tectonics, sequence deposit distribution, and hydrocarbon potential. AAPG Bull. 855 (2001) pp. 885-907.

[6] A. Ben Ferjani, P.F. Burollet, F. Mejri, Petroleum geology of Tunisia. Mémoire ETAP N¹, 1991 $194 p$

[7] W.F. Bishop, Petroleum geology of east - central Tunisia. AAPG Bull. 72 (9), (1988) pp. 10331054.

[8] T. Bralower, Calcareous nannofossils biostratigraphy and assemblages of the CenomanianTuronian boundary interval : implications for origin and timing of oceanic anoxia. Paleoceanography, 3 , (1988) pp. 275-316.

[9] P.F., Burollet, Contribution a l'étude stratigraphique de la Tunisie centrale, Ann. Mines Géol., , n¹8, (1956) 350p.

[10] G. Busson, A. Dhondt, F. Amedro, D. Neraudeau, et A. Cornée, La grande transgression du Cénomanien supérieur-Turonien inférieur sur la Hamada de Tinrhert (Sahara algérien): datations biostratigraphiques, environments de dépôt et comparaison d'un témoin épicratonique avec les séries contemporaines a` matière organique du Maghreb. Cretac. Res. (1999) 20, pp. 29-46.

[11] M. Caron, F. Robaszynski, F. Amédro, F. Baudin, J.-F. Deconinck, P. Hochuli, K. von Salis-Perch Nielsen and N. Tribovillard, Estimation de la durée de l'événement anoxique global au passage Cénomanien/Turonien. Approche cyclostratigraphique dans la Formation Bahloul en Tunisie centrale. Bull. Soc. Géol. Fr. (1999) 170, 2, pp. 145-160.

[12] J. Erbacher et J. Thurrow, Influence of oceanic anoxic events on the evolution of mid-Cretaceous radiolaria in the North Atlantic and western Tethys: Marine Micropaleontology, (1997) - vol. 30, pp. 139-158.

[13] A.S. Gale, H.C., Jenkyns, W.J., Kennedy, R.M., Corfield, Chemostratigraphy versus biostratigraphy: data from around the Cenomanian-Turonian boundary. J. Geol. Soc.. (1993) 150, pp. $29-32$.

[14] W.K., Gealey, Plate tectonic evolution of the Mediterranean- Middle East region. Tectonophysics 155 (1988) pp. 285- 306.

[15] R. Guiraud et J.C. Maurin Le rifting en Afrique au Crétacé inférieur: synthèse structurale, mise en évidence de deux étapes dans la genèse des bassins, relations avec les ouvertures océaniques périafricaines. Bull. Soc. Géol. Fr. (1991) 162, pp. 811-823.

[16] J. Hardenbol, J. Thierry, M.B. Farley, T. Jacquin, P.-C. de Graciansky et P.R. Vail Cretaceous sequence chronostratigraphy. In: P.-C. de Graciansky, J. Hardenbol, T. Jacquin and P.R. Vail, Editors, Mesozoic and Cenozoic Sequence Stratigraphy of European Basins, Soc. Econ. Paleontol. Mineral. Spec. Publ. vol. 60, Chart 4; Tulsa . (1998)

[17] I. Jarvis, G.A. Carson, M.K.E. Cooper, M.B. Hart, et P.N. Leary, B.A. Tocher, D. Horne and A. Rosenfeld Microfossil assemblages and the Cenomanian-Turonian (Late Cretaceous) oceanic anoxic event. Cretac. Res. 9 (1988) pp. 3-103. 
[18] V. Karakitsios, H. Tsikos, Y. van Breugel, L Koletti, J.S. Sinninghe Damsté, , H.C Jenkyns, First evidence for the Cenomanian-Turonian oceanic anoxic event (OAE2, 'Bonarelli' event) from the lonian Zone, western continental Greece. Int. J. Earth Sci., 96, 343-352. (2007)

[19] W. Kuhnt, J.P. Herbin, J., Thurow, J., Wiedmann, Distribution of Cenomanian -Turonian organic facies in the western Mediterranean and along the adjacent Atlantic margin. In: Huc, A.Y. (Ed.), Deposition of Organic Facies. AAPG Studies in Geology, vol. 30, (1990) pp. 133- 160.

[20] M. Layeb Étude géologique, géochimique et minéralogique, régionale, des faciès riches en matière organique de la formation Bahloul d'âge Cénomano-Turonien dans le domaine de la Tunisie Nord-Centrale, Thèse, Doct, Université deTunis. (1990)

[21] A.L. Maâmouri, D. Zaghbib-Turki, M.F. Matmati, M. Chikhaoui, et J. Salaj, La Formation Bahloul en Tunisie centro-septrentionale: variations latérales, nouvelle datation et nouvelle interprétation en terme de stratigraphie séquentielle. J. Afr. Earth Sci. 18 (1) (1994), pp. 37-50.

[22] M. Montacer, J.R. Disnar et J.J. Orgeval, Etude préliminaire de la matière organique de la formation Bahloul dans l'environment sédimentaire du gisement $\mathrm{Zn}-\mathrm{Pb}$ de Bou Grine (Tunisie). Org. Geochem., 13, (1988) pp.423-431

[23] A.J. Nederbragt et A. Fiorentino Stratigraphy and palaeoeoceanography of the CenomanianTuronian Boundary Event in Oued Mellegue, north-western Tunisia. Cretac. Res. 20 (1999) pp. 4762.

[24] F., Robaszynski, M., Caron, C., Dupuis, F., Amedro, J.-M., Gonzalez Donoso, D., Linares, J., Hardenbol, S., Gartner, F., Calandra, and R., Deloffre, A tentative integrated stratigraphy in the Turonian of central Tunisia: formations, zones and sequential stratigraphy in the Kalaat Senan area. Bull. Cent. Rech. Explor. Prod. Elf-Aquitaine 14 (1), (1990) pp. 213-384.

[25] S.O.Schlanger, et H.C. Jenkyns, Cretaceous oceanic anoxia events: causes and consequences. Geol. Mijnb. (1976) 55, pp. 179-184.

[26] S.O., Schlanger, M.A., Arthur, H.C. Jenkyns, et P.A. Scholle, The Cenomanian-Turonian oceanic anoxic event: I. Stratigraphy and distribution of organic-rich beds and the marine ${ }^{13} \mathrm{C}$ excursion. In: J. Brooks and A.J. Fleet, Editors, Marine and Petroleum Source Rocks, Geol. Soc., London, Special Publ. vol. 26 (1987) - pp. 371-399.

[27] M. Soua, Biostratigraphie de haute résolution des foraminifères planctoniques du passage Cénomanien Turonien et impact de l'événement anoxique EAO-2 sur ce groupe dans la marge sud de la Téthys, exemple régions de Jerissa et Bargou. Mémoire de Mastère, Univ., de Tunis El Manar, 2005 169p.

[28] J. Thurow, et W.Kuhnt, Mid-Cretaceous of the Gibraltar Arch area. In: Summerhayes, C.P., Shackleton, N.J. (Eds.), North American Palaeoceanography. Geol. Soc., , Special Publ., vol. 21, (1986) pp. 423-445.

[29] F. Baudin, N. Tribovillard, et J. Trichet, Géologie de la Matière organique. Vuibert, Paris, 263 p. 

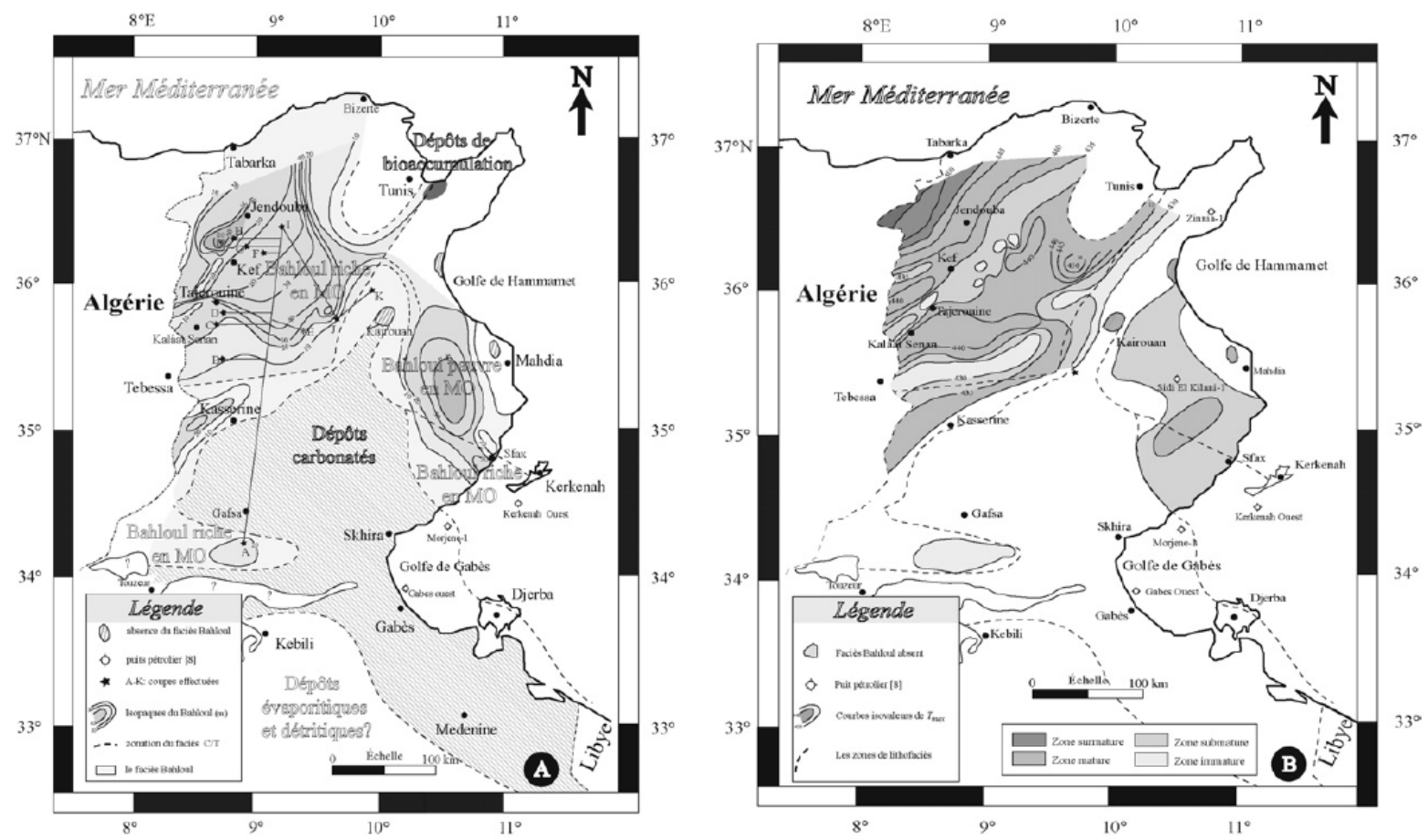

Figure 1 - (A) Les différents faciès et épaisseurs des dépôts de la Formation Bahloul d'âge cénomanien - turonien et leurs équivalents latéraux dans les différents domaines de la Tunisie. La teinte grisée indique la présence de la formation Bahloul, [27]. Noter la coupe A-K (Fig.3) indiquée par un trait continu. (B) Les zonations de maturité de la formation Bahloul [7, 20, 22].

Figure 1 - (A) Different $C / T$ deposit facies, Bahloul isopachs and lateral equivalents in Tunisia. grey colour indicates the Bahloul [27]. Note the A-K section (Fig. 2). (B) Maturity zonation in the Bahloul [7, 20, 22].

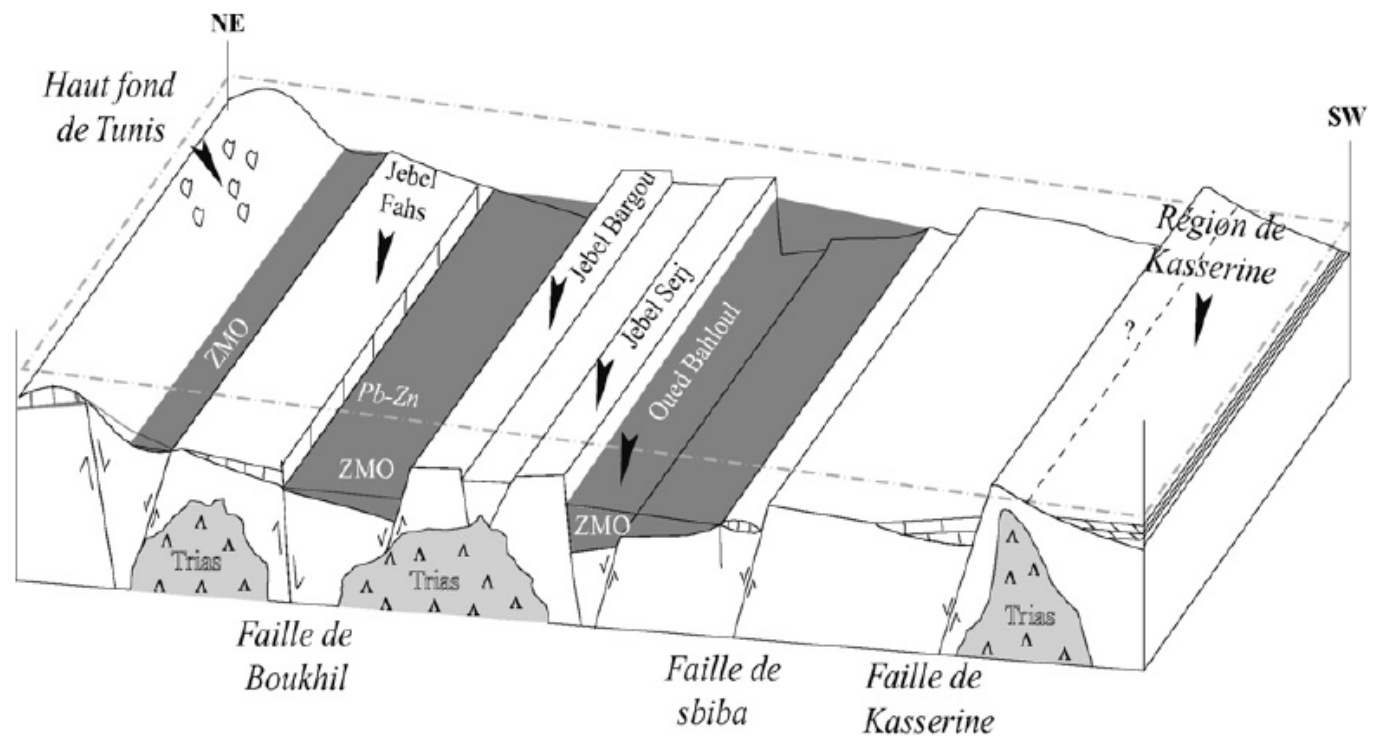

Figure 2 - Paléogéographie au cours de la sédimentation des couches riches en MO contrôlée par la tectonique synsédimentaire du Trias dans les domaines centro-septentrional et centro-méridional. ZMO : zone à minimum d'oxygène

Figure 2 - Paleogeography during organic-rich Bahloul deposition controlled by syndepositional Triassic tectonics. 


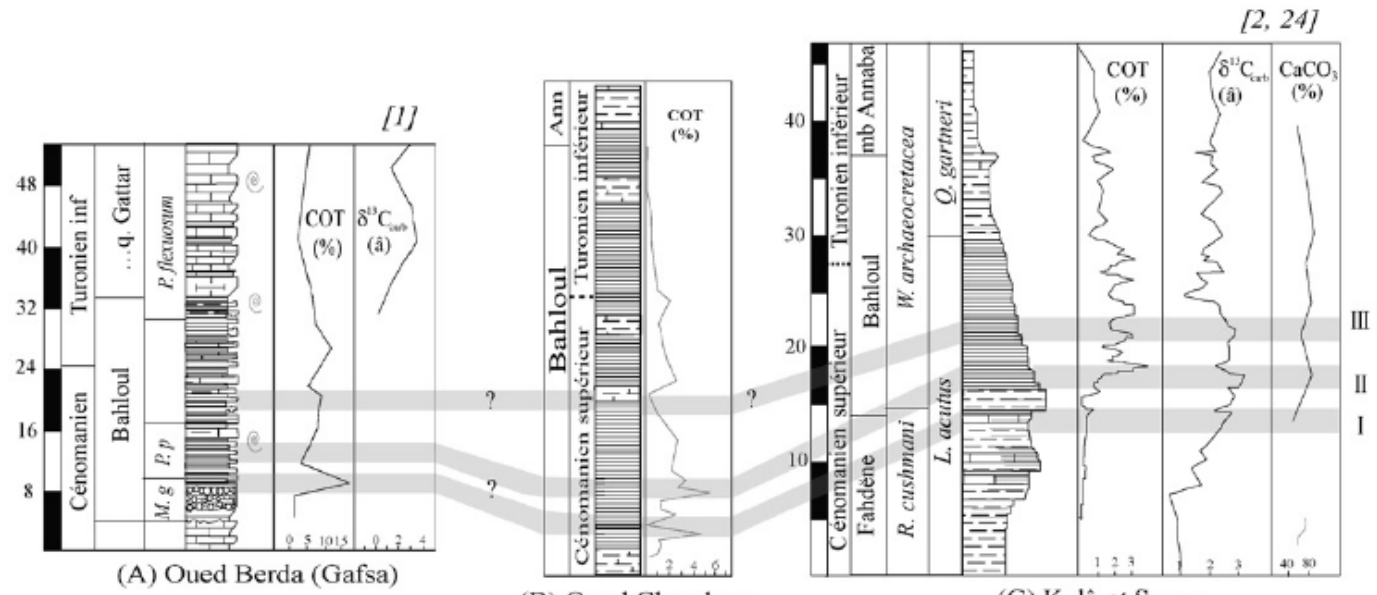

(B) Oued Charchara

(C) Kalâ, at Senan
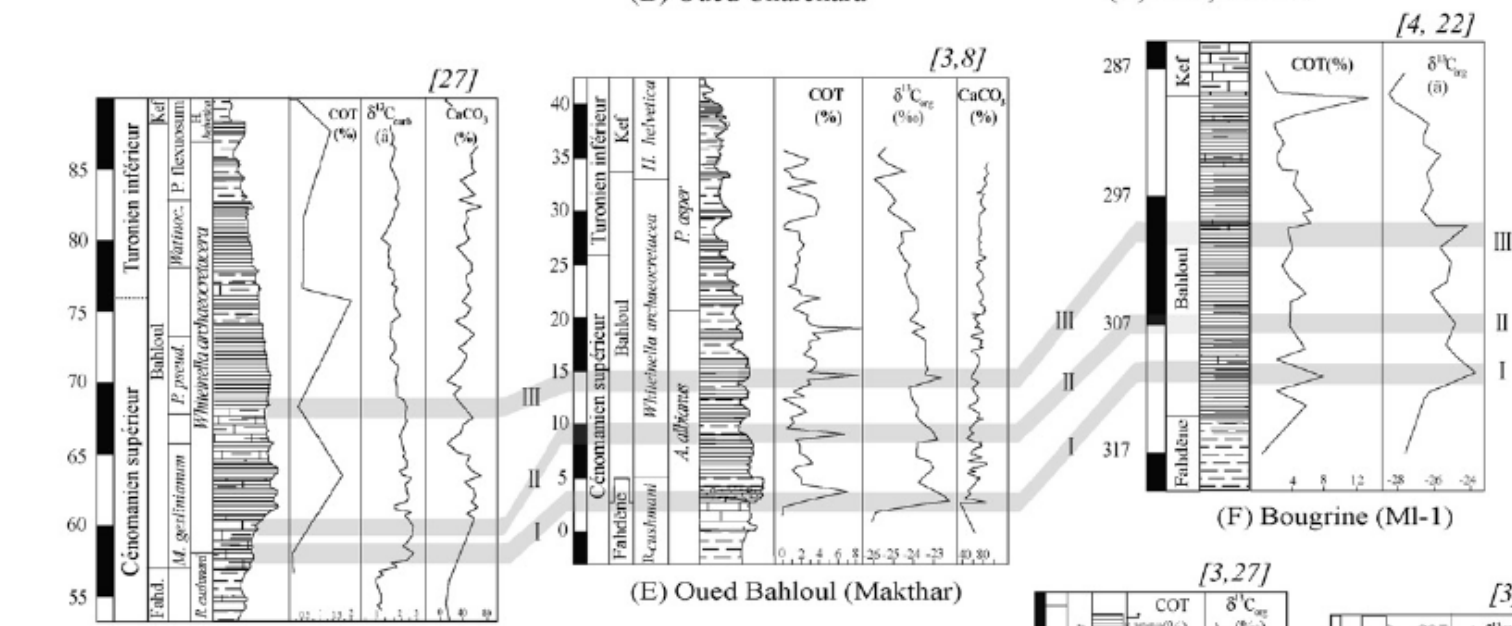

(F) Bougrine (Ml-1)

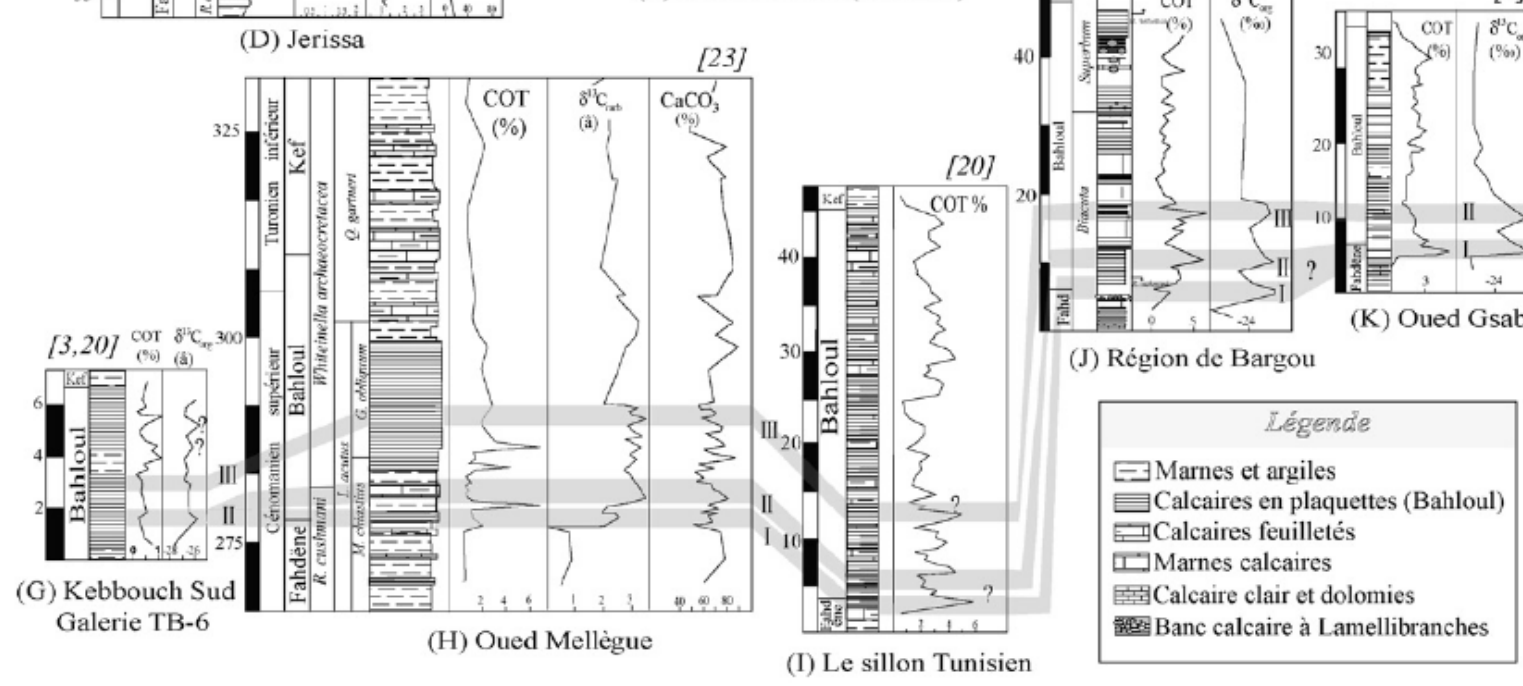

Figure 3 - Essai de corrélation entre les différents domaines de dépôt à partir des profils isotopiques de $\delta^{13} \mathrm{C}$ de COT et de $\mathrm{CaCO}_{3}$. Noter la corrélation entre le changement dans le faciès des sédiments d'âge cénomanien-turonien et les variations des profils isotopiques $\left(\delta^{13} \mathrm{C}\right)$ et la teneur en COT du $S$ vers le $N$ et du NE vers le SW. Les chiffres romains I, II et III indiquent les trois excursions successives du signal isotopique au passage $C / T$.

Figure 3 - High resolution isotopic $\left(\delta^{13} \mathrm{C}\right)$ correlation of the Bahloul. Note the $\mathrm{C} / \mathrm{T}$ facies change and the isotopic variations through the studied transect. I, II and III indicate the three worldwide isotopic excursions occurring near the Cenomanian - Turonian boundary 


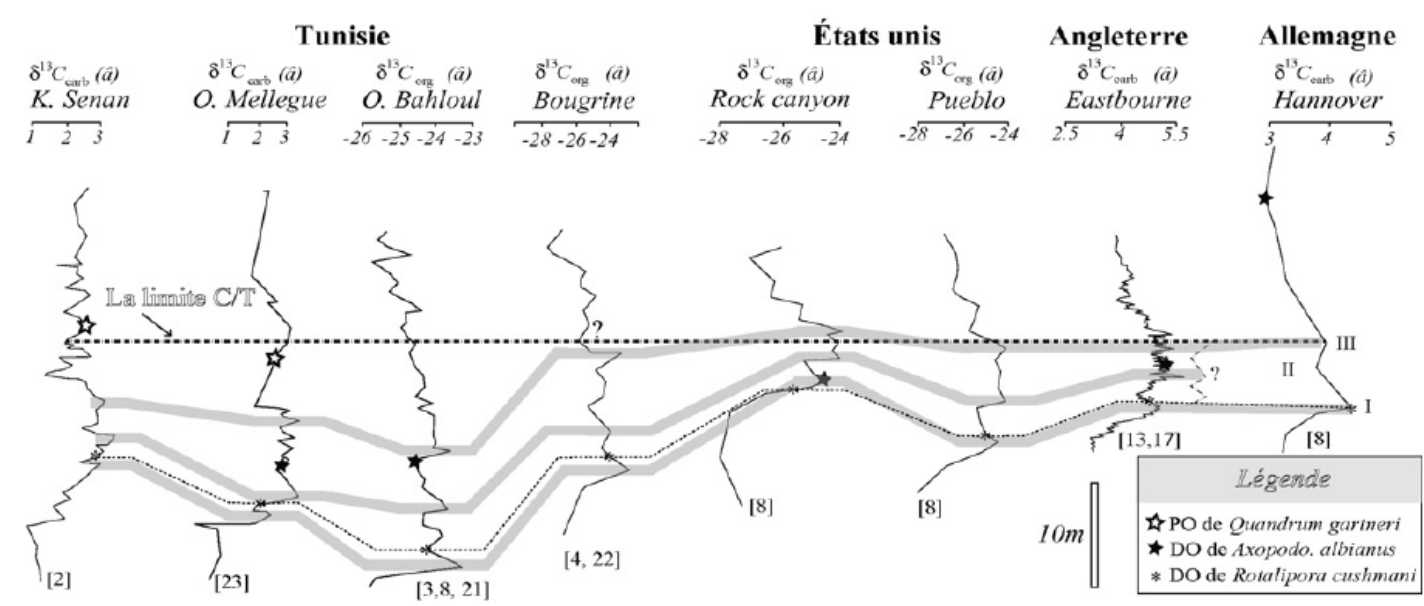

Figure 4 - Comparaison des variations du $\delta^{13} \mathrm{C}$ à l'échelle globale en regard de la position de la limite Cénomanien - Turonien; subdivision isotopique triple (I, II et III); corrélation des premières occurrences $(\mathrm{PO})$ et des dernières occurrences (DO) des nannofossiles et des foraminifères planctoniques.

Figure 4 - Comparison of $\delta^{13} \mathrm{C}$ variations at a global scale with regard to Cenomanian - Turonian boundary position, triple isotopic subdivision (I, II, III), nannofossils and planktonic foraminiferal first (FO) and last occurrences (LO)

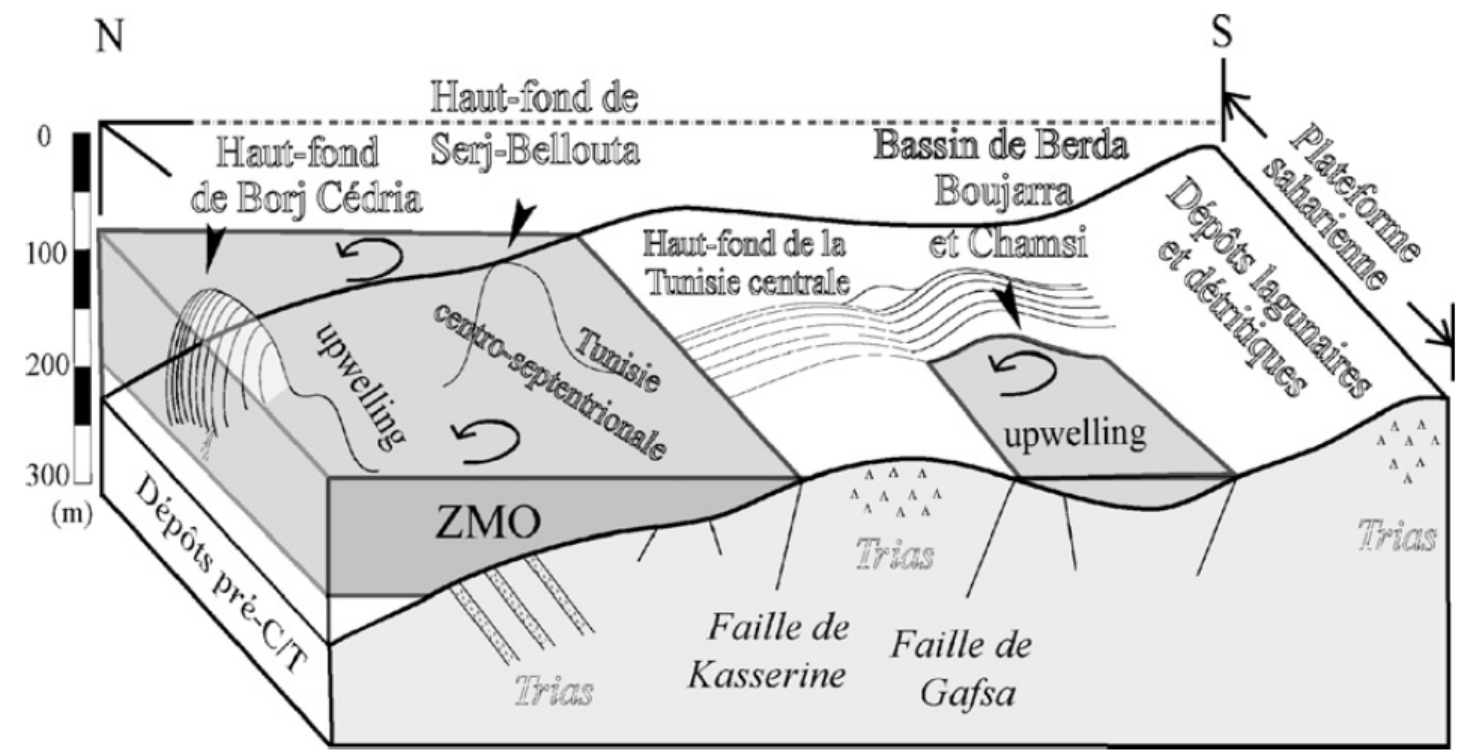

Figure 5 - Modèle de dépôt proposé pour les sédiments riches en MO de la Formation Bahloul en Tunisie. Ces faciès sont impliqués dans une ZOM qui contrôle la distribution des couches riches en MO.Basé partiellement sur les modèles proposés par [7] pour le domaine oriental, [3] pour le domaine centro-septentrional et celui de [1] pour le domaine centro-méridional.

Figure 5 - Proposed depositional model for the organic-rich Bahloul deposits in Tunisia controlled by an impingement of oxygen minimum zone (ZOM). Based partly on [9] for oriental area, [4] for the north-central area and [1] for the south-central area. 